\title{
HYSCORE ON PHOTOEXCITED TRIPLET STATES
}

Claudia E. Tait ${ }^{1,2^{*}}$, Patrik Neuhaus ${ }^{3}$, Harry L. Anderson ${ }^{3}$, Christiane R. Timmel ${ }^{2}$, Donatella Carbonera ${ }^{1}$, Marilena Di Valentin ${ }^{{ }^{*}}$

${ }^{1}$ Dipartimento di Scienze Chimiche, Università degli Studi di Padova, Via Marzolo 1, 35131 Padova, Italy

2Department of Chemistry, Centre for Advanced Electron Spin Resonance, University of Oxford, South Parks Road, Oxford OX1 3QR, United Kingdom

${ }^{3}$ Department of Chemistry, Chemistry Research Laboratory, University of Oxford, 12 Mansfield Road, Oxford OX1 3TA, United Kingdom

*Email: claudia.tait@chem.ox.ac.uk; Tel: +44 1865 272641; Email: marilena.divalentin@unipd.it; Tel: +39 498275139

\begin{abstract}
HYSCORE (Hyperfine Sublevel CORrElation spectroscopy) is one of the most widely applied EPR techniques for the investigation of hyperfine couplings in a wide range of spin systems; here it is applied for the first time to a photoexcited triplet state. The analytical expressions describing the electron spin echo envelope modulations in a HYSCORE experiment on a triplet state coupled to spin $1 / 2$ nuclei and spin 1 nuclei with weak nuclear quadrupole interactions were derived employing the density matrix formalism and used to determine the characteristic features of this experiment when applied to a triplet state. Experimental HYSCORE spectra recorded on the photoexcited triplet state of a meso-substituted zinc porphyrin are used to investigate the ${ }^{14} \mathrm{~N}$ hyperfine interactions in this system. The results are compared to one-dimensional three-pulse ESEEM (Electron Spin Echo Envelope Modulation) experiments and the comparison clearly shows the advantage of the HYSCORE experiment for investigation of the hyperfine couplings of a spin 1 nucleus for triplet states in disordered powder samples. The experimental HYSCORE data are discussed and interpreted in light of the properties of triplet state HYSCORE spectra identified using our theoretical approach.
\end{abstract}

\section{Introduction}

Photoexcited triplet states are of interest in a range of research fields, including natural and artificial photosynthesis [1-9], development of electrophosphorescent organic light-emitting devices [10], photodynamic therapy [11, 12] and, as shown recently, also for protein structure resolution using Double Electron Electron Resonance (DEER) [13]. The characterisation of their electronic structure is of considerable importance for a wide range of applications.

Information on the hyperfine couplings of nuclei to paramagnetic systems can be obtained using ENDOR (Electron Nuclear Double Resonance) and one- and two-dimensional ESEEM (Electron Spin Echo Envelope Modulation) experiments [14]. Photoexcited ENDOR and ESEEM have been extensively applied to the study of a series of triplet states [15-18]; in particular of chlorophylls and carotenoid molecules in proteins involved in photosynthesis, where the results have shed light on energy transfer processes in natural photosynthesis [5, 19-25]. For organic radicals and transition metal centres, the two-dimensional ESEEM experiment HYSCORE has provided important advantages with respect to onedimensional two- and three-pulse ESEEM [14, 2628]. However, to the best of our knowledge, no published account of its application to photoexcited organic triplet state molecules exists.

A theoretical description of ESEEM experiments based on the derivation of analytical expressions describing the electron spin echo envelope modulation function has proven fundamental in the past for the interpretation of experimental data and has provided insight into the main features and characteristics of these experiments [29-31]. Several approaches to a numerical calculation of ESEEM and HYSCORE spectra have been developed and are usually employed for the simulation of experimental data and extraction of the hyperfine and nuclear quadrupole coupling parameters [32-35]. However, analytical expressions, although often only applicable for special cases where simplifying assumptions are possible, can often provide further insight and understanding of the experimental results and help to optimise the experimental conditions for data acquisition. The explicit analytical expressions for the echo modulation in a number of systems and for different pulse sequences have been derived following Mims' density matrix formalism [30]. Specifically, analytical expressions for twopulse and three-pulse ESEEM and HYSCORE have been derived for $S=1 / 2, l=1 / 2$ and $l=1$ systems [30, 36, 37]. As far as $S>1 / 2$ spin systems are concerned, 
expressions for the echo modulation in the twopulse ESEEM experiment have been derived for $S=1, I=1 / 2$ systems [15] and the $S=5 / 2$ case $\left(\mathrm{Mn}^{2+}\right.$ complexes) has been analysed in some detail [3840]. Sloop et al. have derived expressions for the echo modulation in two-pulse ESEEM experiments on $S=1, I=1 / 2$ systems and have found that the triplet state can be approximated to a fictitious $S=1 / 2$ system assuming transition selectivity [15, 16].

In this paper we describe the application of HYSCORE to photoexcited triplet states from a theoretical and an experimental point of view, by extending the derivation of analytical expressions to $S=1, I=1$ systems with weak nuclear quadrupole interactions. The most important features of the HYSCORE experiment as applied to triplet states are discussed based both on the derived expressions and on experimental HYSCORE spectra recorded for the photoexcited triplet state of a meso-substituted porphyrin. We also discuss the advantages of using HYSCORE rather than two- or three-pulse ESEEM for the determination of hyperfine couplings of spin 1 nuclei, such as ${ }^{14} \mathrm{~N}$, in triplet state systems.

\section{Experimental}

[5,15-Bis(3,5(trihexylsilyl)phenyl)-10,20-bis ((trihexylsilyl)ethynyl)porphyrinato] zinc(II) (see Fig. 3A
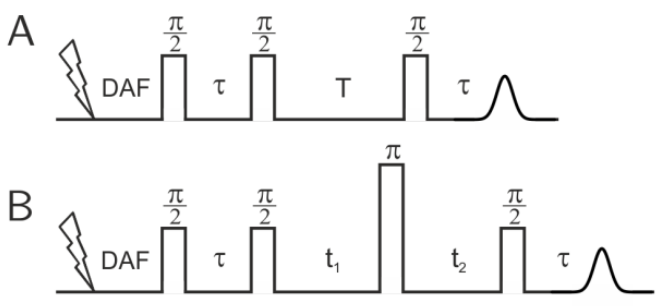

for the structure) was synthesised according to the published procedure [41]. Samples for EPR measurements were prepared in MeTHF:pyridine 10:1 and degassed using several freeze-pump thaw cycles.

$X$-band three-pulse ESEEM and HYSCORE experiments were performed on an X-band Bruker Elexsys 680 spectrometer equipped with a helium gas-flow cryostat from Oxford instruments. Qband HYSCORE measurements were performed on a Bruker Elexsys $580 \mathrm{Q} / \mathrm{S}$-band spectrometer with a 3W TWT amplifier. Photoexcitation with a $10 \mathrm{~Hz}$ $\mathrm{Nd}$ :YAG laser at $532 \mathrm{~nm}$ with a power of $15 \mathrm{~mJ}$ was used to generate the photoexcited triplet state. The experiments were performed at $20 \mathrm{~K}$.

X-band HYSCORE experiments were performed using the pulse sequence shown in Fig. $1 \mathrm{~B}$ with $\mathrm{mw}$ pulses of lengths of $t_{\pi / 2}=16 \mathrm{~ns}, t_{\pi}=16 \mathrm{~ns}$ and $\tau=120,160$ and $200 \mathrm{~ns}$, and starting times $t_{1}=t_{2}=64$ ns with increments $d t=16$ ns (data matrix 256x256). ${ }^{14} \mathrm{~N}$ matched HYSCORE experiments were performed with matched pulses instead of the second and last pulse of the standard HYSCORE sequence. The matched pulses were applied at high microwave power and the pulse lengths were optimised for maximum modulation depth in three-pulse ESEEM experiments with varying pulse lengths (optimum length $=24 \mathrm{~ns}$ ).

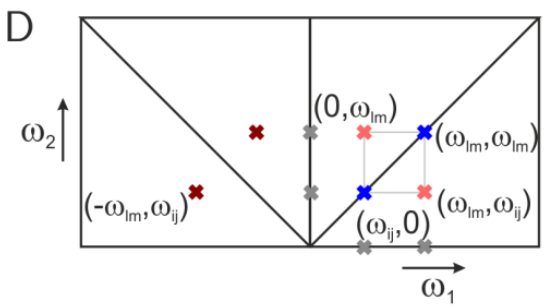

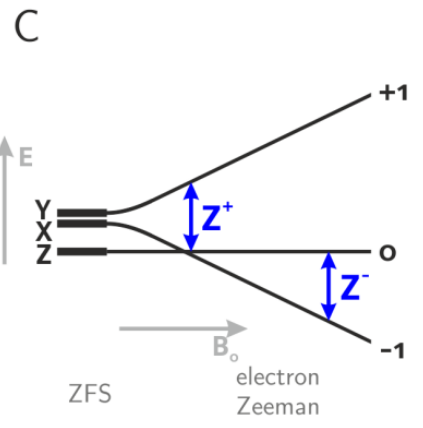
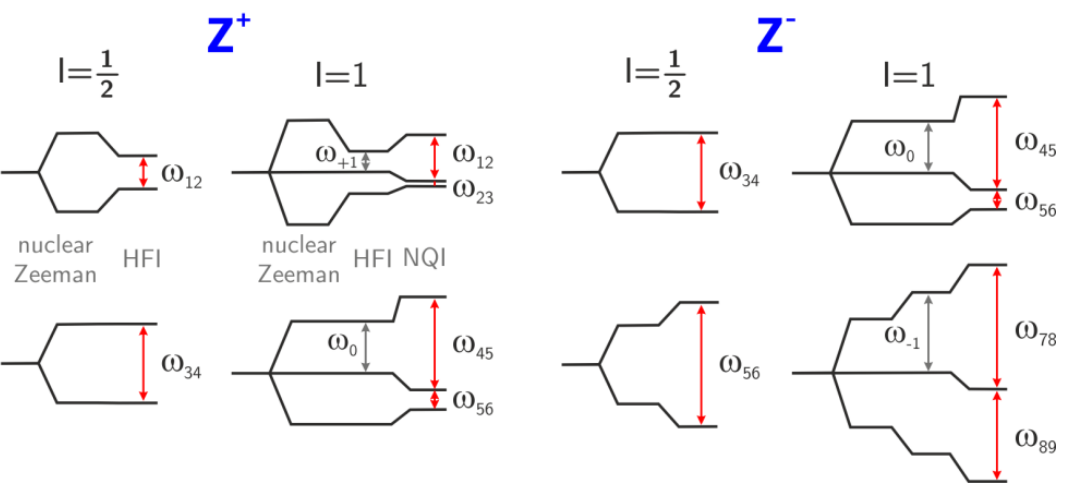

Fig.1. (A, B) Pulse sequences for the three-pulse ESEEM (A) and HYSCORE (B) experiment with laser excitation (DAF = delay after flash). (C) Energy level schemes for a triplet state with $D, E>0$ coupled to a nucleus with either $I=1 / 2$ or $I=1$ with hyperfine coupling $A>0$ and close to the cancellation regime in the case of $l=1$. The magnetic field is aligned with the $Z$ axis of the ZFS tensor. The EPR transitions and the single-quantum frequencies contributing to the ESEEM/HYSCORE modulations for the low- and high-field transition of the triplet state are highlighted in blue and red respectively. (D) Schematic drawing of the upper quadrants of a HYSCORE spectrum showing the possible types of peaks: cross-peaks in different quadrants for weak and strong coupling (light and dark red), diagonal peaks (blue) and axial peaks (grey). 
Q-band HYSCORE was performed with pulse lengths of $t_{\pi / 2}=30 \mathrm{~ns}, t_{\pi}=30 \mathrm{~ns}$ and $\tau=100$ and 212 ns, and starting times $t_{1}=t_{2}=64$ ns with increments $d t=16$ ns (data matrix 256x256).Q-band matched HYSCORE was performed at the $Z$ - field position with 74 ns matched pulses and 8 ns increments, $\tau$ values of 100 and 160 ns were used. A 4-step phase cycle was used to remove unwanted echoes. The experimental data were processed in Matlab; the time traces were baseline corrected by a $3^{\text {rd }}$ order polynomial, apodised with a Hamming window and zero-filled to 1024 points in each dimension. A 2D Fourier transform was applied and the absolute-value spectrum was calculated.

X-band three-pulse ESEEM experiments were performed with the pulse sequence shown in Fig. $1 \mathrm{~A}$ with mw pulses of lengths of $t_{\pi / 2}=16 \mathrm{~ns}$ and an initial T value of $64 \mathrm{~ns}$ with increments $d t=8 \mathrm{~ns}$ (1024 data points were collected). A 4-step phase cycle was used to remove unwanted echoes. The ESEEM traces were recorded for three different $\tau$ values (100 ns, $160 \mathrm{~ns}$ and $200 \mathrm{~ns}$ ) and the FT spectra were summed to avoid distortions due to blind spots. The experimental data were processed with a home-written Matlab program, the time traces were baseline corrected with a stretched exponential, apodised with a Hamming window and zero-filled to 2048 data points. The frequency spectrum was calculated using the cross-term averaging procedure implemented in Easyspin [42, 43].

The analytical expressions describing the time domain of the HYSCORE experiments were derived in Mathematica. The calculated HYSCORE time domain data were processed as described above to yield the HYSCORE spectra in the frequency domain.

\section{Theory}

The complete analytical expressions describing the four-pulse HYSCORE experiment for an $S=1$ system coupled to $I=1 / 2$ and $I=1$ nuclei are derived here based on the density matrix formalism, first introduced for the analysis of ESEEM by Rowan, Hahn and Mims [29] and later generalised and described in detail by Mims [30].

The spin Hamiltonian for a triplet state coupled to a nucleus with arbitrary spin / expressed in angular frequency units is:

$$
\begin{array}{r}
\hat{H}_{0}=\omega_{S} S_{z}+D\left(\hat{S}_{z}^{2}-\frac{1}{3} \hat{S}^{2}\right)+E\left(\hat{S}_{X}^{2}-\hat{S}_{Y}^{2}\right) \\
-\omega_{I} I_{z}+\hat{S} A \hat{I}+\hat{I} P \hat{I}
\end{array}
$$

where the different terms stand for the electron Zeeman interaction with an isotropic $g$-value in the high-field approximation, the zero-field splitting (ZFS) interaction, the nuclear Zeeman interaction, the hyperfine interaction between the electron spin and a nuclear spin $I$ and the nuclear quadrupole interaction, present for a nucleus with $1>1 / 2$. Schematic energy level diagrams for a triplet state coupled to an $I=1 / 2$ or $I=1$ nucleus are shown in Fig. $1 \mathrm{C}$.

A series of simplifying assumptions was necessary to derive the analytical expressions. First of all, the derivations were based on the high-field approximation for the electron spin and ideal pulses were considered throughout. Further, since the spacing of the triplet state sublevels due to zero-field splitting is generally such that the microwave frequency cannot simultaneously excite transitions that share a common sublevel, transition selective microwave pulses were considered. In this case, the triplet system can be well approximated to a fictitious spin $1 / 2$ system under the conditions that $g B_{\mathrm{e}} B_{1}<<D$, where $B_{1}$ is the amplitude of the microwave field and $D$ is the zero-field splitting parameter [15, 38].

The modulation formula for the HYSCORE experiment, describing the modulation of the echo amplitude with time in two dimensions, was previously derived for the $S=1 / 2 \quad I=1 / 2$ system by Gemperle et al. [36] and later revised by Tyryshkin et al. [44]. It is usually written as a sum of two contributions to emphasize the different evolution pathways of the nuclear spins: $E_{\bmod }^{\alpha \beta}\left(E_{\bmod }^{\beta \alpha}\right)$ describes the nuclear spins precessing with the frequencies of the $\alpha(\beta)$ manifold during $t_{1}$ and of the $\beta(\alpha)$ manifold during $t_{2}$. Here we extend their results by calculating the equivalent expressions for the $S=1, I=1 / 2$ and $S=1, l=1$ systems.

\subsection{Systems with $S=1$ and $I=1 / 2$}

The modulation formula for the $m_{\mathrm{s}}=0$ to $m_{\mathrm{s}}=+1$ transition in an $S=1, l=1 / 2$ system is:

$$
\begin{aligned}
& E_{\bmod }\left(\tau, t_{1}, t_{2}\right)=\frac{1}{2}\left[E_{\bmod }^{\alpha \beta}\left(\tau, t_{1}, t_{2}\right)+E_{\bmod }^{\beta \alpha}\left(\tau, t_{1}, t_{2}\right)\right] \\
& E_{\bmod }^{\alpha \beta}\left(\tau, t_{1}, t_{2}\right)= \\
& \chi_{0}+\chi_{d}+ \\
& \gamma_{-}\left[\chi_{12} \cos \left(\omega_{12} t_{1}+\frac{\omega_{12} \tau}{2}\right)\right. \\
& +\chi_{12} \cos \left(\omega_{34} t_{2}+\frac{\omega_{34} \tau}{2}\right) \\
& +\chi_{12,34}^{+} \cos \left(\omega_{12} t_{1}+\omega_{34} t_{2}+\frac{\left(\omega_{12}+\omega_{34}\right) \tau}{2}\right) \\
& \left.+\chi_{12,34}^{-} \cos \left(\omega_{12} t_{1}-\omega_{34} t_{2}+\frac{\left(\omega_{12}-\omega_{34}\right) \tau}{2}\right)\right]
\end{aligned}
$$


$E_{\text {mod }}^{\beta \alpha}$ is obtained by exchanging $t_{1}$ and $t_{2}$ in the expression for $E_{\bmod }^{\alpha \beta}$. The $\chi$ coefficients are defined in the appendix. An analogous expression is obtained for the second triplet state transition between $m_{s}=0$ and $m_{s}=-1$. The results differ from those obtained for the corresponding $S=1 / 2$ system by the definition of the nuclear frequencies and the modulation depth parameters. The nuclear frequencies $\omega_{12}\left(m_{s}=+1\right), \omega_{34}\left(m_{s}=0\right)$ and $\omega_{56}\left(m_{s}=-\right.$ 1) (in angular frequency units) are obtained by diagonalisation of the nuclear spin subHamiltonian [14, 30]:

$$
\hat{H}_{0}=-\omega_{I} I_{z}+A S_{z} I_{z}+B S_{z} I_{x}
$$

Leading to the nuclear frequencies:

$$
\begin{aligned}
& \omega_{12}=\omega_{+1}=\sqrt{\left(\omega_{I}-A\right)^{2}+B^{2}} \\
& \omega_{34}=\omega_{0}=\omega_{I} \\
& \omega_{56}=\omega_{-1}=\sqrt{\left(\omega_{I}+A\right)^{2}+B^{2}}
\end{aligned}
$$

In case of an axially symmetric hyperfine interaction, the coefficients $A$ and $B$ are related to the isotropic hyperfine constant $a_{i s o}$ and to the dipolar hyperfine coupling $A_{d i p}=[-T,-T, 2 T]$ as

$$
\begin{aligned}
& A=a_{i s o}+T\left(3 \cos ^{2} \theta-1\right) \\
& B=3 T \sin \theta \cos \theta
\end{aligned}
$$

The expression in equation 2 contains several different terms leading to different types of peaks in the HYSCORE spectrum (see Fig. 1D):

\section{$\chi_{0}$ is an unmodulated term;}

- $\quad \chi_{d}$ gives rise to peaks along the diagonal ( $\omega_{\mathrm{ij}}$, $\left.\omega_{\mathrm{ij}}\right)$ for each nuclear frequency if the mixing pulse deviates from an ideal $\pi$ pulse (see below);

- terms of the type $\chi_{i j} \cos \left(\omega_{i j} t_{1 / 2}+\frac{\omega_{i j} \tau}{2}\right)$ give rise to axial peaks $\left(\omega_{i j}, 0\right) /\left(0, \omega_{i j}\right)$, which are, however, usually eliminated by the baseline correction [14];

- terms of the
$\chi_{i j, l m}^{+-l} \cos \left(\omega_{i j} t_{1} \pm \omega_{l m} t_{2}+\frac{\left(\omega_{i j} \pm \omega_{l m}\right) \tau}{2}\right)$ give rise to cross-peaks $\left( \pm \omega_{\mathrm{ij}}, \omega_{\mathrm{Im}}\right)$ in the $(+,+)$ or $(+,-)$ quadrant of the HYSCORE spectrum.

The positions of the cross-peaks allow determination of the nuclear frequencies and hence of the hyperfine couplings between electron and nuclear spin. As can be seen from equation 2 , for an $S=1, I=1 / 2$ system only two cross-peaks are expected in each quadrant.

The intensities of the different peaks in the HYSCORE spectrum depend on the coefficients $\chi$, which depend on the modulation depth parameter $k$. The modulation depth parameters $k_{+1 /-1}$ for the two triplet state transitions are defined as:

$$
k_{+1 /-1}=\left(\frac{B}{\omega_{12 / 56}}\right)^{2}
$$

eq. 6

The differences in the modulation depth parameters are due to the dependence on the nuclear frequencies of the manifolds connected by the transition. In analogy to $S=1 / 2$ systems, modulations are only observed in the presence of an anisotropic hyperfine interaction.

In general, cross-peaks can appear in any of the quadrants of the HYSCORE spectrum and are usually observed in the $(+,+)$ quadrant for weak hyperfine couplings and in the $(-,+)$ quadrant for strong hyperfine couplings (see Fig. 1D). The terms in the modulation formula accounting for peaks in the two quadrants are weighted by terms of the form $\sqrt{1-k_{+1 /-1}} \pm 1$ (see expressions for $\chi_{i j, l m}^{+/-}$in the appendix). Due to the different definitions of the modulation depth parameter $k_{+1 /-1}$ for the two triplet transitions, the relative intensities of peaks in the two quadrants will be different. The modulation depth parameter only depends on the hyperfine coupling and the nuclear frequency of the $m_{\mathrm{s}}=+1\left(\omega_{12}\right)$ or the $m_{\mathrm{s}}=-1\left(\omega_{56}\right)$ manifold (see equation 6). Since the modulation depth parameter will be larger for the transition involving the smaller of the two nuclear frequencies, peaks in the $(-,+)$ quadrant will be stronger for the corresponding transition with respect to the transition involving the other two manifolds. For example, for a positive hyperfine coupling the nuclear frequency of the $m_{s}=+1$ manifold $\left(\omega_{12}\right)$ is smaller than the nuclear frequency for the $m_{s}=-1$ manifold $\left(\omega_{56}\right)$ and hence $k_{+1}>k_{-1}$. The HYSCORE spectrum for the $m_{\mathrm{s}}=0$ to $m_{\mathrm{s}}=+1$ transition will thus have more intense peaks in the $(-,+)$ quadrant with respect to the spectrum for the $m_{s}=-1$ to $m_{s}=0$ transition. The shift of the signals from the $(+,+)$ quadrant to the $(-,+)$ quadrant with increasing hyperfine interaction will also occur at different points.

\subsection{Systems with $S=1$ and $I=1$}

An analytical diagonalisation of the nuclear subHamiltonians for $I=1$, comprising nuclear Zeeman, hyperfine and nuclear quadrupole interaction, is of 
considerable difficulty. If the nuclear quadrupole interaction can be assumed small with respect to the nuclear Zeeman and hyperfine interaction, as is generally legitimate for $I=1$ nuclei except ${ }^{14} \mathrm{~N}$ [45], a simplified treatment can be used, in which the effect of the nuclear quadrupole interaction is taken into account in the nuclear frequencies, but neglected in the derivation of the modulation expression. If such an approach is taken, then the nuclear frequencies may be determined by diagonalisation of the nuclear sub-Hamiltonians without the nuclear quadrupole interaction and its consequent incorporation through first-order perturbation theory, yielding the following results:

$$
\begin{array}{ll}
\omega_{12}=\omega_{+1}+\Delta & \omega_{23}=\omega_{+1}-\Delta \\
\omega_{45}=\omega_{0}+\Delta & \omega_{56}=\omega_{0}-\Delta \\
\omega_{78}=\omega_{-1}+\Delta & \omega_{89}=\omega_{-1}-\Delta
\end{array}
$$

where $\omega_{+1}, \omega_{0}$ and $\omega_{-1}$ are defined in eq. 4 .

The correction to the energy values for an axially symmetric nuclear quadrupole interaction is [37, 45, 46]:

$$
\Delta_{m_{I}}=\frac{e^{2} q Q}{4 I(2 I-1)}\left(\frac{3 \cos ^{2} \theta-1}{2}\right)\left[3 m_{t}^{2}-I(I+1)\right] \quad \text { eq. } 8
$$

with $\theta$ as the angle between the nuclear quadrupole tensor and the effective magnetic field $\vec{B}_{\text {eff }}$ interacting with the nucleus (where $\vec{B}_{e f f}=\vec{B}_{0}+\vec{B}_{h f}$ with $\vec{B}_{h f}$ as the field generated at the nucleus by the hyperfine interaction with the electron spin).

The HYSCORE modulation expression for the transition between the $m_{s}=0$ and $m_{s}=+1$ manifold in an $S=1, I=1$ system derived in this manner yields the following result:

$$
\begin{aligned}
& E_{\bmod }\left(\tau, t_{1}, t_{2}\right)=\frac{1}{2}\left[E_{\bmod }^{\alpha \beta}\left(\tau, t_{1}, t_{2}\right)+E_{\bmod }^{\beta \alpha}\left(\tau, t_{1}, t_{2}\right)\right] \\
& E_{\bmod }^{\alpha \beta}\left(\tau, t_{1}, t_{2}\right)= \\
& \chi_{0}+\chi_{d}+ \\
& \gamma_{-}\left[\sum_{i=1}^{3} \sum_{j>i}^{3} \chi_{i j} \cos \left(\omega_{i j} t_{1}+\frac{\omega_{i j} \tau}{2}\right)\right. \\
& +\sum_{l=4}^{6} \sum_{m>l}^{6} \chi_{l m} \cos \left(\omega_{l m} t_{2}+\frac{\omega_{l m} \tau}{2}\right) \\
& +\sum_{i=1}^{3} \sum_{j>i}^{3} \sum_{l=4}^{6} \sum_{m>l}^{6} \chi_{i j, l m}^{+} \cos \left(\omega_{i j} t_{1}+\omega_{l m} t_{2}+\frac{\left(\omega_{i j}+\omega_{l m}\right) \tau}{2}\right) \\
& \left.+\sum_{i=1}^{3} \sum_{j>i}^{3} \sum_{l=4}^{6} \sum_{m>l}^{6} \chi_{i j, l m}^{-} \cos \left(\omega_{i j} t_{1}-\omega_{l m} t_{2}+\frac{\left(\omega_{i j}-\omega_{l m}\right) \tau}{2}\right)\right] \\
& \quad \text { eq. } 9
\end{aligned}
$$

where $E_{\bmod }^{\beta \alpha}$ is obtained by exchanging $t_{1}$ and $t_{2}$ in the expression for $E_{\text {mod }}^{\alpha \beta}$. The coefficients $\chi$ are defined in the appendix.

A comparison of equation $9(I=1)$ with equation 2 $(I=1 / 2)$ shows that they contain the same type of terms leading to the same type of HYSCORE peaks as discussed for the simpler $S=1, I=1 / 2$ case. However, since there are now two single-quantum and one double-quantum nuclear frequencies for each electron spin manifold, the number of crosspeaks increases from two to 18 in each quadrant.

The cross-peak intensities for single crystal ${ }^{2} \mathrm{H}$ HYSCORE spectra for an $S=1 / 2$ system were discussed from a theoretical and experimental point of view in reference [37]. The authors showed that 16 of the cross-peak terms arise from $\Delta m_{1}= \pm 1$ nuclear transitions and 20 cross-peaks from combinations of nuclear frequencies involving the $\Delta m_{1}= \pm 2$ nuclear transitions, but that the intensity of most of them is too low to be detected experimentally. A comparison of the modulation factors $\chi_{i j, l m}$ of different cross-peaks (given in the appendix) allowed the identification of only two $\left(\chi_{12,45}^{+}\right.$and $\left.\chi_{23,56}^{+}\right)$, which depend linearly on the modulation depth parameter $k$, as observed also in the $S=1 / 2$ case [37]. Hence, in analogy to the $S=1 / 2$ case, only four of the eight theoretically possible cross-peaks arising in the first quadrant from $\Delta m_{1}= \pm 1$ nuclear transitions relative to a single EPR transition are experimentally observable, namely the cross-peaks at $\left(\omega_{12}, \omega_{45}\right),\left(\omega_{45}, \omega_{12}\right),\left(\omega_{23}, \omega_{56}\right)$ and $\left(\omega_{56}, \omega_{23}\right)$. The intensities of the double-quantum cross-peaks $\left(\omega_{13}, \omega_{46}\right)$ and $\left(\omega_{46}, \omega_{13}\right)$ depend on the square of the modulation depth parameter and are thus predicted to be weaker.

The terms defining the intensities for signals in the $(+,+)$ and $(-,+)$ quadrants are more complicated in this case, however they follow the same trends explained for the simpler $l=1 / 2$ systems, i.e. the peaks in the $(-,+)$ quadrant are stronger for the triplet transition with the smaller nuclear frequency and the shift of the signals from the $(+,+)$ to the $(-,+)$ quadrant will occur at different hyperfine couplings, depending on the relative magnitude of the nuclear frequencies of the $m_{\mathrm{s}}=+1$ or $m_{s}=-1$ manifold compared to the nuclear Larmor frequency.

In the application of the HYSCORE pulse sequence to an $S>1 / 2$ centre, complications can arise due to the different nominal pulse angle with respect to the simpler $S=1 / 2$ case, since the nominal pulse angle depends on the $m_{\mathrm{s}}$ quantum numbers of the 
manifolds of the selected EPR transition [40]. In the case of a triplet state system both allowed EPR transitions require the same nominal pulse angle; the effective rotation for an $S=1$ system corresponds to $\sqrt{2}$ times the rotation for an $S=1 / 2$ system [15]. In case of incomplete inversion by the mixing pulse, diagonal peaks appear and the intensity of the cross-peaks is reduced, as can be seen from the multiplicative factors $\gamma_{+}$and $\gamma$ - in equations 2 and 9.

$$
\gamma_{ \pm}=\left(1 \pm \sec \sqrt{2} \omega_{N} t_{p I I I}\right) \quad \text { eq. } 10
$$

where $\omega_{N}$ stands for the strength of the $B_{1}$ field and $t_{p l l l}$ is the length of the third (mixing) pulse. If complete inversion is assumed, the terms in the echo modulation expression giving rise to diagonal peaks are absent. Experimentally the length of this pulse can be optimised for complete inversion in order to reduce diagonal peaks.

The derivation of an analytical expression for HYSCORE of a nuclear spin $l=1$ with strong nuclear quadrupole interaction using this method was not possible. Analytical treatments of nuclear spin $l=1$ systems are usually only possible under severely simplifying assumptions or for special cases [40, $47,48]$. Different alternatives used to describe the powder patterns generated in HYSCORE spectra based on the Cayley-Hamilton theorem or on catastrophe theory have also been proposed [49, 50]. Nevertheless it is often necessary to resort to either numerical simulations, such as implemented in GAMMA [32, 34] or Matlab [35, 43] or consideration of just the frequency positions of the cross-peaks, as the relative intensities are often difficult to reproduce [26].

\section{Results and Discussion}

Pulse EPR of triplet state systems is usually strongly orientation-selective, as the microwave pulses only excite a small fraction of the EPR spectrum typically broadened significantly by the zero-field splitting interaction. Hence only a limited number of orientations contribute to the consequently often single-crystal like ENDOR, ESEEM and HYSCORE spectra. In the most general case of $0<E<D / 3$ this is strictly true for the outermost ( $\mathrm{Z}$ ) transitions, while more orientations contribute to the $X$ and $Y$ transitions. In the special case of $E=D / 3$ the $Z$ and $Y$ transitions coincide and orientations of the molecule with either of these axes aligned with the magnetic field are excited, while the orientation selection for $\mathrm{X}$ is similar to the general case. In the case of an axial ZFS tensor $(E=0)$, pure orientation selection is again obtained for the $Z$ canonical transition, while the orientations on the whole plane perpendicular to $\mathrm{Z}$ are excited for the other canonical transition. Hence in this case single-crystal like spectra are only obtained for the $\mathrm{Z}$ orientation, unless the hyperfine tensor is also axial and collinear with the ZFS tensor. However, in all other cases even if multiple orientations are excited for the $X$ and $Y$ canonical transitions, typically the orientations lying along the ZFS tensor axes contribute preferentially to the hyperfine spectrum due to increased relaxation for non-canonical orientations [51], leading to a larger distribution with respect to the $\mathbf{Z}$ transition, but smaller still compared to the absence of orientation selection. In the case of the hyperfine techniques, this means that measurements close to the canonical positions, corresponding to the three axes of the ZFS tensor, can be considered to a good approximation to reflect the hyperfine couplings along those directions in the molecular frame. HYSCORE spectra of triplet state systems are thus expected to exhibit single peaks instead of the broad ridges typical of many $S=1 / 2$ systems in most cases.

In addition to orientation-selection, triplet state pulse EPR is also characterised by transition selection, i.e. only the transition between two of the triplet state sublevels is selected depending on the magnetic field position. HYSCORE spectra hence only contain nuclear frequencies of two of the three manifolds $\left(m_{s}=+1\right.$ and $m_{s}=0$, or $m_{s}=-1$ and $m_{s}=0$ ). Both contain the nuclear frequencies of the $m_{s}=0$ manifold, determined exclusively by the nuclear Zeeman and nuclear quadrupole interaction (for $I=1$ ) (see Fig. 1C). In triplet state ENDOR this transition selectivity allows the determination of the sign of the hyperfine coupling, if the sign of the ZFS parameter $D$ is known [52]. It will be shown that the sign of the hyperfine couplings can also be determined using triplet state HYSCORE.

\subsection{Theoretical Results}

Fig. 2 shows HYSCORE spectra obtained by 2D FFT of electron spin echo envelope modulation data calculated using equations 2 and 9 for triplet states coupled to a spin $1 / 2$ or a spin 1 nucleus and for a single orientation of the spin system with respect to the magnetic field.

In the case of spin $1 / 2$ nuclei, such as for example ${ }^{1} \mathrm{H}$, only two cross-peaks are observed for each of the two triplet state transitions corresponding to a single orientation of the molecule with respect to the external magnetic field. The cross-peaks are given by $\left(\mathrm{v}_{12}, \mathrm{v}_{34}\right)$ and $\left(\mathrm{v}_{34}, \mathrm{v}_{56}\right)$ (frequencies in $\mathrm{MHz}$ ), where for a positive hyperfine coupling $v_{12}<v_{34}<$ $v_{56}$, while for a negative hyperfine coupling the 


\section{$S=1 \quad I=\frac{1}{2}$}
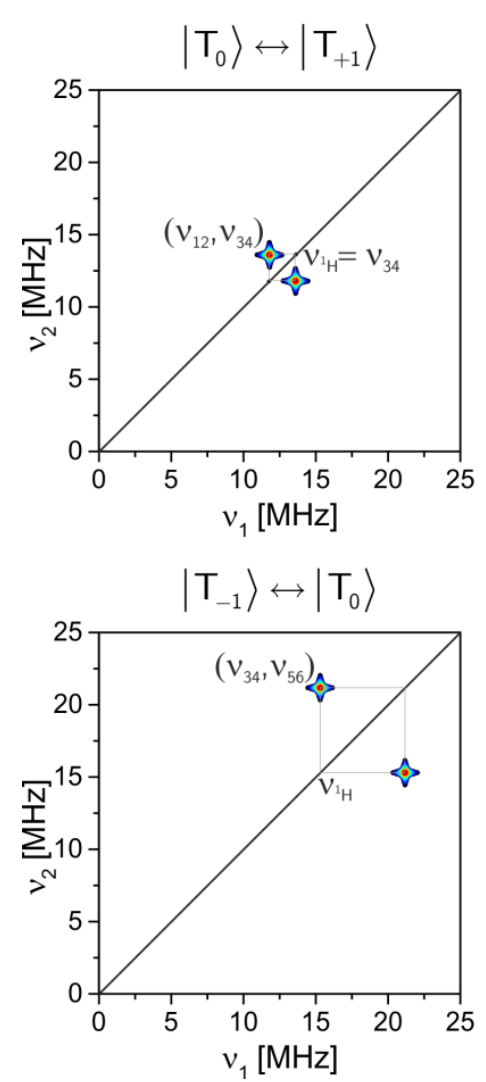

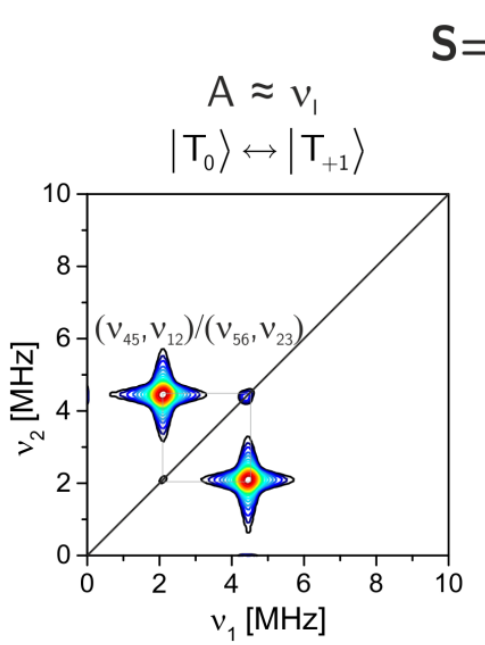

$S=1 \quad I=1$
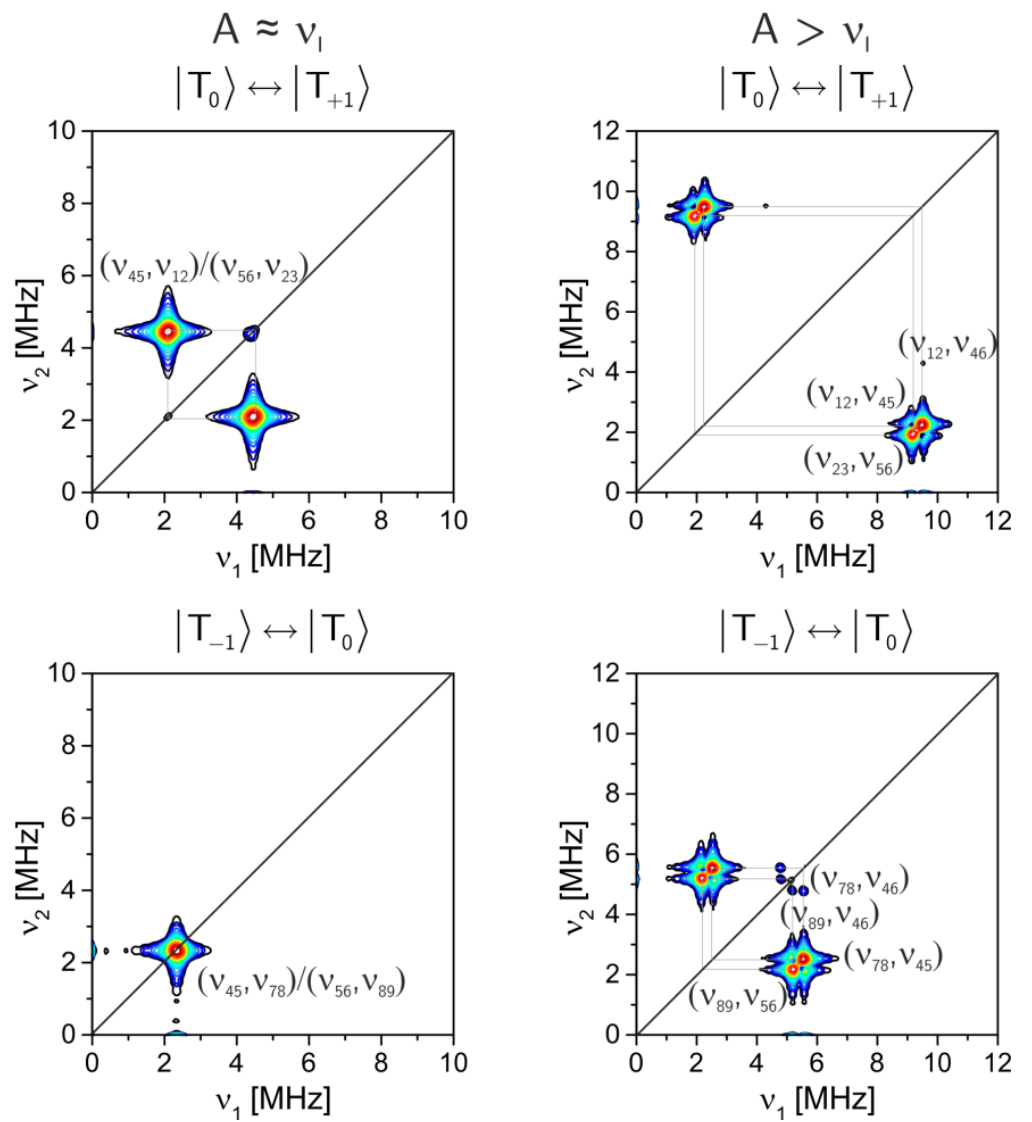

Fig.2. Calculated HYSCORE spectra for the two triplet transitions in an $S=1, l=1 / 2$ system with $v_{l}=13.6 / 15.3 \mathrm{MHz}, a_{\text {iso }}=+2 \mathrm{MHz}$, $T=+5 \mathrm{MHz}$ and $\theta=45^{\circ}$ (left) and for an $S=1, l=1$ system with $v_{l}=2.09 / 2.35 \mathrm{MHz}$ close to cancellation regime $\left(a_{\text {iso }}=-1 \mathrm{MHz}, T=-\right.$ $1.5 \mathrm{MHz}, \theta=45^{\circ}$ and $Q=0.25 \mathrm{MHz}$ ) (centre) and with $A>v_{1}\left(a_{\text {iso }}=-2 \mathrm{MHz}, T=-3 \mathrm{MHz}, \theta=22^{\circ}\right.$ and $Q=0.25 \mathrm{MHz}$ ) (right). The frequencies corresponding to the cross-peaks are indicated based on the definitions given in the text. The other quadrant of the HYSCORE spectra is not depicted as the cross peaks are absent or much weaker.

order is reversed. The position of the cross-peaks with respect to the nuclear Larmor frequency can thus be used to determine the sign of the hyperfine coupling. If more than a single orientation were selected, there would be a distribution of nuclear frequencies in the $m_{s}=-1$ and $m_{s}=+1$ manifolds, but not in the $m_{s}=0$ manifold, leading to ridges parallel to the frequency axes. This is a specific feature of triplet state HYSCORE.

For spin 1 nuclei with weak nuclear quadrupole couplings spectra were calculated for a hyperfine coupling comparable to (cancellation regime, Fig. 2 centre) or larger than the nuclear Zeeman frequency $v_{1}$ (Fig. 2 right). In the cancellation regime $\left(A \approx v_{1}\right.$ for a triplet state) in addition to the nuclear frequencies of the $m_{s}=0$ manifold, the nuclear frequencies of one of the other manifolds also do not depend on the hyperfine coupling and reduce to the pure nuclear quadrupole frequencies $\left(m_{s}=-1\right.$ for $A<0$ and $m_{s}=+1$ for $\left.A>0\right)$. The nuclear frequencies of the two manifolds will be very similar in many cases, one depending only on the nuclear quadrupole interaction and the other on the nuclear quadrupole and nuclear Zeeman interaction, and hence a single peak on or close to the diagonal will be observed in the HYSCORE spectrum for that transition (see Fig. 2, bottom centre). All the information on the hyperfine coupling is in this case contained in the HYSCORE spectrum of the triplet state transition involving the other $m_{\mathrm{s}}$ manifold, for which intense crosspeaks are observed (see Fig. 2, top centre).

If the hyperfine coupling is larger than $v_{1}$, at least four cross-peaks are observed for each of the transitions, at positions determined by the singlequantum frequencies. In this case of weak nuclear quadrupole coupling, the double-quantum crosspeaks are predicted to have negligible intensity. In the presence of a distribution of hyperfine and nuclear quadrupole splittings due to selection of more than one orientation again ridges approximately parallel to the axes are expected, since the changes in hyperfine coupling are usually 

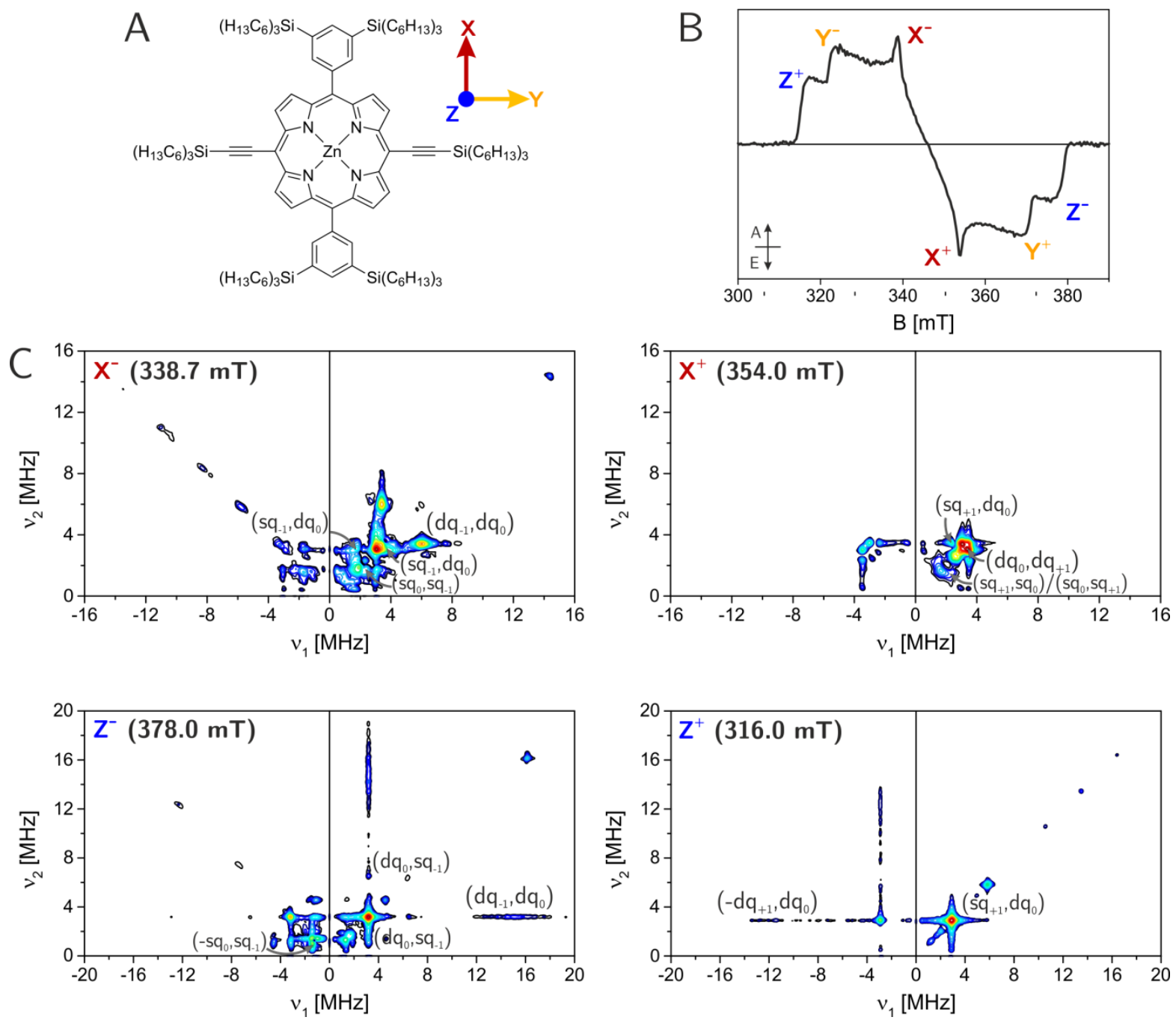

Fig.3. (A) Molecular structure of the investigated zinc porphyrin with orientation of the ZFS tensor with respect to the molecular frame. (B) X-band time-resolved EPR spectrum of the photoexcited triplet state of the molecule shown in (A) recorded in MeTHF:Pyr 10:1 and averaged up to $2 \mu \mathrm{s}$ after laser excitation at $532 \mathrm{~nm}(D=890 \mathrm{MHz}, E=155 \mathrm{MHz}$, $A=$ absorption, $E=e m i s s i o n)$. (C) Experimental $X$-band ${ }^{14} \mathrm{~N}$ HYSCORE spectra recorded for the two triplet state transitions in the canonical X (in-plane) and Z (out-of-plane) orientations. The spectra at $338.7 \mathrm{mT}, 354.0 \mathrm{mT}$, and $316.0 \mathrm{mT}$ were recorded using standard HYSCORE and the spectrum at $378.0 \mathrm{mT}$ was recorded using matched HYSCORE. The experimental parameters are defined in the experimental section. The main cross peaks in the HYSCORE spectra are tentatively assigned to the nuclear frequencies of the different triplet manifolds.

more significant with respect to those of the nuclear quadrupole interaction.

The simulations shown in Fig. 2 were performed assuming ideal pulses, leading to the absence of artefact peaks along the diagonal, as discussed in section 3. Experimentally this is often difficult to achieve and peaks along the diagonal with varying intensity compared to the cross-peaks will be observed.

\subsection{Experimental Results}

${ }^{14} \mathrm{~N}$ HYSCORE spectra of the zinc porphyrin depicted in Fig. $3 \mathrm{~A}$ were recorded at field positions corresponding to orientations along the in-plane $X$ and out-of-plane Z ZFS axes after laser excitation at $532 \mathrm{~nm}$ and are shown in Fig. 3C. Measurements along the second in-plane axis $Y$ gave similar spectra as along $X$, indicating an approximately axial hyperfine tensor. ${ }^{14} \mathrm{~N}$ nuclei in porphyrin-like systems are typically characterised by a nuclear quadrupole coupling of about 2-3 $\mathrm{MHz}[19,53]$ and the assumption of weak nuclear quadrupole coupling is no longer valid. For $S=1 / 2$ systems, I=1 nuclei with weak nuclear quadrupole coupling $\left({ }^{2} \mathrm{H}\right)$ were shown to give strong singlequantum peaks in the HYSCORE spectrum and weak double-quantum peaks, as also predicted here for the triplet state. On the other hand, $l=1$ nuclei with strong nuclear quadrupole coupling interactions such as ${ }^{14} \mathrm{~N}$ give rise to HYSCORE spectra with strong double-quantum features and 
weaker single-quantum peaks [28]. This implies that the intensities of the HYSCORE features due to the ${ }^{14} \mathrm{~N}$ nuclei will not be accurately predicted by equation 9; however a discussion of the theoretical and experimental data side-by side is very instructive as it shall be shown that despite its shortcomings and approximations, the theoretical considerations above predict many features observed experimentally in this porphyrin system.

The strongest cross-peaks observed in the ${ }^{14} \mathrm{~N}$ HYSCORE spectra have been attributed to doublequantum cross-peaks. Double-quantum frequencies do not depend on the nuclear quadrupole coupling to first order and can be used to obtain an estimate of the hyperfine couplings [28]. In almost all of the spectra strong peaks on the diagonal are observed, which are mostly due to non-ideal mixing pulses.

The HYSCORE spectra recorded at field positions corresponding to the two triplet state transitions for the $X$ direction of the ZFS tensor $\left(X^{+}\right.$and $\left.X^{-}\right)$ exhibit strong cross-peaks at about $(6.1,3.2) \mathrm{MHz}$ and $(3.2,6.1) \mathrm{MHz}$ for $X^{-}$(cross-peaks marked as $\left.\left(\mathrm{dq}_{-1}, \mathrm{dq}_{0}\right)\right)$ and cross-peaks very close to the diagonal for $\mathrm{X}^{+}$(cross-peaks marked as $\left(\mathrm{dq}_{0}, \mathrm{dq}_{+1}\right)$ ). This resembles what was observed in the simulations for weak nuclear quadrupole coupling in the exact cancellation regime (Fig. 2 centre). In
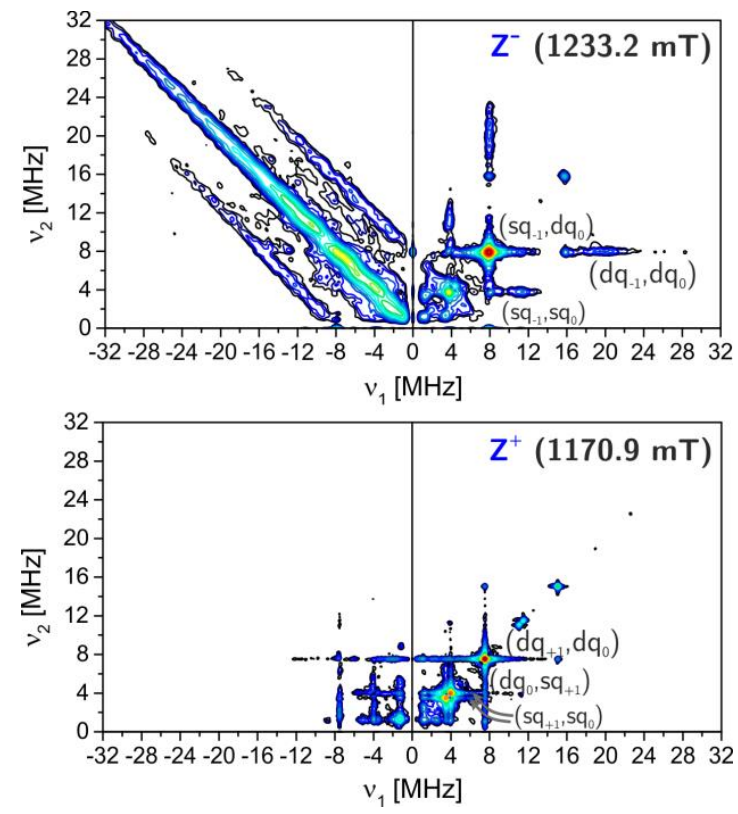

Fig.4. Experimental Q-band ${ }^{14} \mathrm{~N}$ HYSCORE (bottom) and matched HYSCORE (top) spectra recorded at the high and low field $\mathrm{Z}$ field positions. The experimental parameters are described in the experimental section. The main cross peaks in the HYSCORE spectra are tentatively assigned to the nuclear frequencies of the different triplet manifolds. this regime the difference between the two double-quantum frequencies approximately corresponds to two times the hyperfine coupling for that orientation; based on calculations the error of neglecting the contribution of the nuclear quadrupole coupling amounts to an underestimate of about $20 \%$. The in-plane hyperfine coupling for the pyrrolic nitrogen nuclei can thus be estimated to be about $1.7 \mathrm{MHz}$. The sign of the hyperfine coupling is positive, since the larger nuclear frequencies are observed for the $X^{-}$transition and the $D$ value of the porphyrin molecule is positive (see Fig. 1).

The HYSCORE spectra recorded for the out-ofplane orientation are more difficult to interpret. First of all, the double-quantum cross-peaks for the $Z^{-}$transition could only be clearly resolved using matched HYSCORE, which leads to an increase in the intensity of multiple quantum features [54]. Secondly, instead of single-crystallike spectra, broad ridges parallel to the frequency axes were observed. The hyperfine coupling estimated from the centre of these ridges is about 6.5 MHz, which is far from the cancellation regime where HYSCORE spectra are easier to interpret.

The HYSCORE measurements for the out-of-plane orientation were thus repeated at Q-band, closer to the cancellation regime, and the resulting spectra are shown in Fig. 4. In this case the ridges for $\mathrm{Z}^{-}$are observed more clearly, while for $\mathrm{Z}^{+}$they are closer to the diagonal, confirming that the

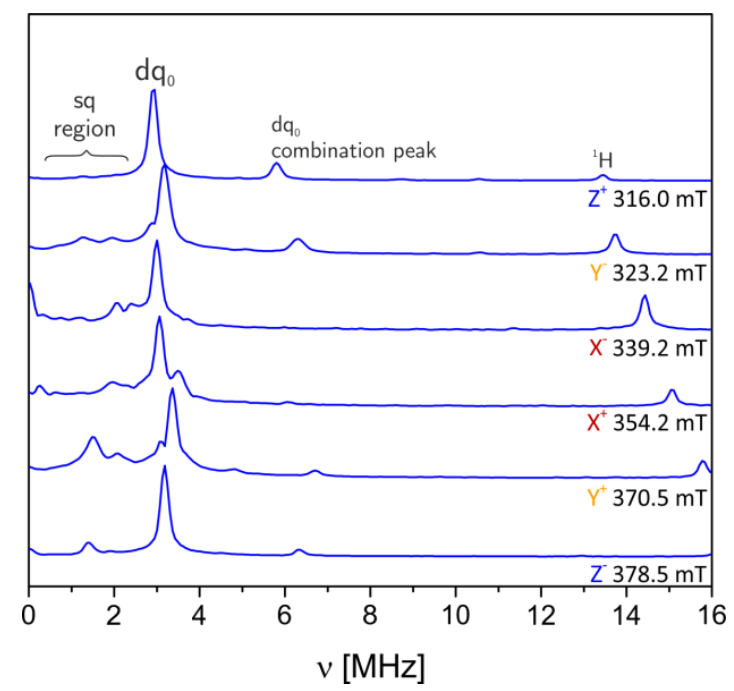

Fig.5. Experimental $\mathrm{X}$-band ${ }^{14} \mathrm{~N}$ three-pulse ESEEM spectra recorded in MeTHF at the six canonical field positions highlighted in the EPR spectrum shown in Fig. $3 B$. The main peaks were tentatively assigned to the nuclear frequencies of the $\mathrm{m}_{\mathrm{s}}=0$ manifold (see text). 

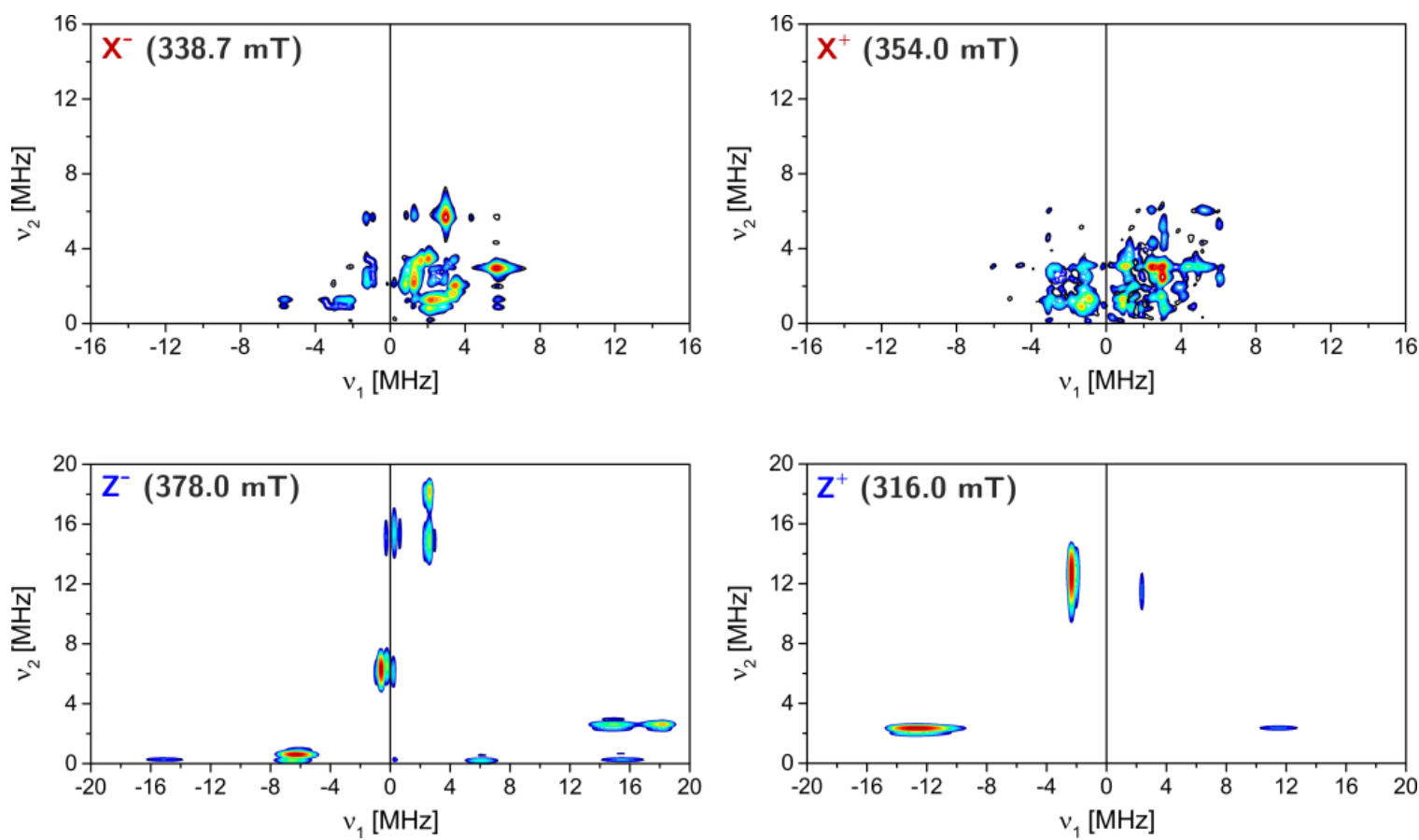

Fig.6. Simulations of the ${ }^{14} \mathrm{~N}$ HYSCORE spectra of the zinc porphyrin performed using Easyspin's saffron function [35, 43] with the following hyperfine and nuclear quadrupole parameters: $A_{x}=1.55 \pm 0.15 \mathrm{MHz}, A_{\mathrm{y}}=1.65 \pm 0.15 \mathrm{MHz}, A_{z}=6.95 \pm 1.35$ $\mathrm{MHz}, \mathrm{Q}=2.35 \pm 0.05 \mathrm{MHz}$ and $\eta=0.75 \pm 0.10$.

average hyperfine coupling is about $6.5 \mathrm{MHz}$ and has a positive sign. The observation of ridges instead of single-crystal-like peaks expected on account of orientation selection, which should be strongest for the $\mathrm{Z}$ orientation, was attributed to a distribution in hyperfine couplings, in analogy to the results in previous papers $[53,55]$. The origin for the distribution might be the conformational flexibility of the porphyrin plane leading to changes in hyperfine couplings, particularly in the out-of plane orientation [56]. The coordinated pyridine molecule was identified as a possible cause for distortions of the porphyrin plane and a closer analysis of the solvent interaction and the influence of pyridine on the ESEEM and HYSCORE data will be the subject of further investigation.

The X-band three-pulse ESEEM spectra recorded for the six canonical field positions are shown in Fig. 5. The spectra are dominated by the doublequantum nuclear frequency of the $\mathrm{m}_{\mathrm{s}}=0$ manifold; some peaks in the low frequency region are also present, but their exact assignment was difficult. The signals corresponding to the double-quantum nuclear frequencies of the other two manifolds are too broad to be clearly identified due to the distribution of hyperfine couplings. The inhomogeneous hyperfine broadening that affects the line shapes in three-pulse ESEEM is refocused by the mixing pulse in HYSCORE, achieving better resolution and allowing the detection of broad peaks [14]. The nitrogen hyperfine couplings could not have been unambiguously determined from the ESEEM data alone; the HYSCORE data with the clearly resolved double-quantum features were essential for the characterisation of the investigated porphyrin system in terms of the ${ }^{14} \mathrm{~N}$ hyperfine interaction.

The nitrogen hyperfine and nuclear quadrupole parameters were determined by simulation of the HYSCORE spectra. Since no analytical solutions could be derived for ${ }^{14} \mathrm{~N}$ nuclei, numerical simulations implemented in Easyspin [35, 43] were employed. The HYSCORE spectra were simulated for a set of hyperfine and nuclear quadrupole parameters simultaneously optimised for X- and Qband HYSCORE and three-pulse ESEEM data. The simulation parameters were $A_{x}=1.55 \pm 0.15 \mathrm{MHz}$, $A_{\mathrm{y}}=1.65 \pm 0.15 \mathrm{MHz}, \quad A_{\mathrm{z}}=6.95 \pm 1.35 \mathrm{MHz}$, $Q=2.35 \pm 0.05 \mathrm{MHz}$ and $\eta=0.75 \pm 0.10$, where the standard deviations indicate the width of the distribution around the central values considered in order to simulate the observed ridges. The tensor orientations were defined based on DFT calculations [56]. The resulting simulated HYSCORE spectra are shown in Fig. 6. The agreement between simulation and experiment is satisfyingly close; in particular the peak positions are well reproduced. The strong peaks on the diagonal in the experimental data are absent in the simulations, confirming that they are due to incomplete inversion by the mixing $\pi$ pulse. The simulated relative intensities of the different types of cross peaks also agree quite well with experiment, considering that an accurate 
simulation of HYSCORE peak intensities can often be challenging $[26,34]$.

\section{Conclusions}

The features of HYSCORE spectra of photoexcited triplet states coupled to spin $1 / 2$ and spin 1 nuclei were discussed based on the analytical expressions derived in the framework of the density matrix formalism. It was shown that the orientation and transition selection in these systems allows the determination of the hyperfine coupling in different orientations of the molecular frame. In addition to this, the sign of the hyperfine coupling can also be determined, assuming the sign of the zero-field splitting parameter $D$ is known. The conclusions drawn based on example calculations were used to interpret experimental HYSCORE spectra of the triplet state of a porphyrin molecule.

\section{Acknowledgements}

The Q-band EPR measurements were performed at the National EPR Research Facility at the University of Manchester with the help of Dr. Alistair Fielding. The authors are grateful to Dr. Jeffrey Harmer for helpful discussions and useful comments on the manuscript. The authors gratefully acknowledge the financial support of this work by EPSRC.

\section{References}

[1] R. Uphaus, J. Norris, J. Katz, Triplet states in photosynthesis, Biochem. Biophys. Res. Commun., 61 (1974) 1057-1063.

[2] H. Levanon, J. Norris, The photoexcited triplet state and photosynthesis, Chem. Rev., 78 (1978) 185-198.

[3] D. Budil, M. Thurnauer, The chlorophyll triplet state as a probe of structure and function in photosynthesis, Biochim. Biophys. Acta, 1057 (1991) 1-41.

[4] H. Levanon, K. Möbius, Advanced EPR spectroscopy on electron transfer processes in photosynthesis and biomimetic model systems, Annu. Rev. Biophys. Biomol. Struct., 26 (1997) 495-540.

[5] W. Lubitz, F. Lendzian, R. Bittl, Radicals, Radical Pairs and Triplet States in Photosynthesis, Acc. Chem. Res., 35 (2002) 313-320.

[6] D. Carbonera, M. Di Valentin, R. Spezia, A. Mezzetti, The Unique Photophysical Properties of the PeridininChlorophyll-a-Protein, Current Protein and Peptide Science, 15 (2014) 332-350.

[7] D. Gust, T. Moore, A. Moore, Mimicking photosynthetic solar energy transduction, Acc. Chem. Res., 34 (2001) 40-48.

[8] M. Wasielewski, Energy, charge, and spin transport in molecules and self-assembled nanostructures inspired by photosynthesis, J. Org. Chem., 71 (2006) 5051-5066.

[9] S. Fukuzumi, K. Ohkubo, T. Suenobu, Long-Lived Charge Separation and Applications in Artificial Photosynthesis, Acc. Chem. Res., 47 (2014) 1455-1464.

[10] L. Xiao, Z. Chen, B. Qu, J. Luo, S. Kong, Q. Gong, J. Kido, Recent Progresses on Materials for
Electrophosphorescent Organic Light-Emitting Devices, Adv. Mater., 23 (2011) 926-952.

[11] K. Plaetzer, B. Krammer, J. Berlanda, F. Berr, T. Kiesslich, Photophysics and photochemistry of photodynamic therapy: fundamental aspects, Lasers in medical science, 24 (2009) 259-268.

[12] J. Zhao, W. Wu, J. Sun, S. Guo, Triplet photosensitizers: from molecular design to applications, Chem. Soc. Rev., 42 (2013) 5323-5351.

[13] M. Di Valentin, M. Albertini, E. Zurlo, M. Gobbo, D. Carbonera, Potential Spin Label for Nanometer Distance Measurements by PELDOR Spectroscopy, J. Am. Chem. Soc., 136 (2014) 6582-6585.

[14] A. Schweiger, G. Jeschke, Principles of Pulse Electron Paramagnetic Resonance, Oxford University Press, Oxford, 2001.

[15] D.J. Sloop, H.-L. Yu, T.S. Lin, S.I. Weissman, Electron spin echoes of a photoexcited triplet: Pentacene in $p$ terphenyl crystals, J. Chem. Phys., 75 (1981) 3746-3757.

[16] H.-L. Yu, D.J. Sloop, S.I. Weissman, J. Lin, J. Norris, M.K. Bowman, Electron spin echo modulation of the photoexcited triplets of anthracene in $p$-terphenyl crystals, J. Phys. Chem., 86 (1982) 4287-4290.

[17] T.S. Lin, Electron Spin Echo Spectroscopy of Organic Triplets, Chem. Rev., 84 (1984) 1-15.

[18] W. Buma, E.J.J. Groenen, J. Schmidt, R. de Beer, An electron spin-echo envelope modulation study of the lowest triplet state of pyridine- $d_{5}$ : Spin-density distribution and structure, J. Chem. Phys., 91 (1989) 6549-6566

[19] D.J. Singel, W.A.J.A. van der Poel, J. Schmidt, J.H. van der Waals, R. De Beer, Complete determination of ${ }^{14} \mathrm{~N}$ hyperfine and quadrupole interactions in the metastable triplet state of free-base porphin via electron spin echo envelope modulation, J. Chem. Phys., 81 (1984) 5453-5461.

[20] A. De Groot, R. Evelo, A.J. Hoff, R. De Beer, H. Scheer, Electron Spin Echo Envelope Modulation (ESEEM) Spectroscopy of the triplet state of the primary donor of ${ }^{14} \mathrm{~N}$ and ${ }^{15} \mathrm{~N}$ bacterial photosynthetic reaction centers and of ${ }^{14} \mathrm{~N}$ and ${ }^{15} \mathrm{~N}$ bacteriochlorophyll $a$, Chem. Phys. Lett., 118 (1985) 48-54.

[21] F. Lendzian, R. Bittl, W. Lubitz, Pulsed ENDOR of the photoexcited triplet states of bacteriochlorophyll $a$ and of the primary donor P865 in reaction centers of Rhodobacter sphaeroides R-26, Photosynth. Res., 55 (1998) 189-197.

[22] J. Niklas, T. Schulte, S. Prakash, M. van Gastel, E. Hofmann, W. Lubitz, Spin-Density Distribution of the Carotenoid Triplet State in the Peridinin-ChlorophyllProtein Antenna. A Q-Band Pulse Electron-Nuclear Double Resonance and Density Functional Theory Study, J. Am. Chem. Soc., 129 (2007) 15442-15443.

[23] E. Salvadori, M. Di Valentin, C.M.W. Kay, A. Pedone, V. Barone, D. Carbonera, The electronic structure of the lutein triplet state in plant light-harvesting complex II, Phys. Chem. Chem. Phys., 14 (2011) 12238-12251.

[24] M. Di Valentin, E. Salvadori, V. Barone, D. Carbonera, Unravelling electronic and structural requisites of triplet-triplet energy transfer by advanced electron paramagnetic resonance and density functional theory, Mol. Phys., 111 (2013) 2914-2932.

[25] A. Marchanka, W. Lubitz, M. Plato, M. van Gastel, 
Comparative ENDOR study at $34 \mathrm{GHz}$ of the triplet state of the primary donor in bacterial reaction centers of $R b$. sphaeroides and BI. viridis, Photosynth. Res., 120 (2014) 99-111.

[26] S. van Doorslaer, Hyperfine Sublevel Correlation Spectroscopy, EPR newsletter, 17 (2008) 9-12.

[27] C. Calle, A. Sreekanth, M.V. Fedin, J. Forrer, I. Garcia-Rubio, I.A. Gromov, D. Hinderberger, B. Kasumaj, P. Léger, B. Mancosu, G. Mitrikas, M.G. Santangelo, S. Stoll, A. Schweiger, R. Tschaggelar, J. Harmer, Pulse EPR Methods for Studying Chemical and Biological Samples Containing Transition Metals, Helv. Chim. Acta, 89 (2006) 2495-2521.

[28] J. Harmer, G. Mitrikas, A. Schweiger, Advanced Pulse EPR Methods for the Characterization of Metalloproteins, in: G. Hanson, L. Berliner (Eds.) High Resolution EPR, Springer Science, 2009, pp. 13-61.

[29] L.G. Rowan, E.L. Hahn, W.B. Mims, Electron-SpinEcho Envelope Modulation, Phys. Rev., 137 (1965) A61A71.

[30] W.B. Mims, Envelope Modulation in Spin-Echo Experiments, Phys. Rev. B, 5 (1972) 2409-2419.

[31] W.B. Mims, Amplitudes of Superhyperfine Frequencies Displayed in the Electron-Spin-Echo Envelope, Phys. Rev. B, 6 (1972) 3543-3545.

[32] J.J. Shane, L.P. Liesum, A. Schweiger, Efficient Simulation of ESEEM Spectra Using Gamma, J. Magn. Reson., 134 (1998) 72-75.

[33] R. Szosenfogel, D. Goldfarb, Simulations of HYSCORE spectra obtained with ideal and non-ideal pulses, Mol. Phys., 95 (1998) 1295-1308.

[34] Z. Madi, S. Van Doorslaer, A. Schweiger, Numerical simulation of one- and two-dimensional ESEEM experiments, J. Magn. Reson., 154 (2002) 181-191.

[35] S. Stoll, R.D. Britt, General and efficient simulation of pulse EPR spectra, Phys. Chem. Chem. Phys., 11 (2009) 6614-6625.

[36] C. Gemperle, G. Aebli, A. Schweiger, R.R. Ernst, Phase cycling in Pulse EPR, J. Magn. Reson., 88 (1990) 241-256.

[37] A. Pöppl, R. Böttcher, Cross peak intensities in twodimensional four-pulse electron spin echo modulation spectra of deuteriums in single crystals, Chem. Phys., 221 (1997) 53-66.

[38] A.R. Coffino, J. Peisach, Nuclear Modulation Effects in High-Spin Electron Systems with small Zero-Field Splittings, J. Chem. Phys., 97 (1992) 3072-3091.

[39] R.G. Larsen, C.J. Halkides, D.J. Singel, A geometric representation of nuclear modulation effects - The effects of High Electron-Spin Multiplicity on the Electron-Spin-Echo- Envelope Modulation Spectra of $\mathrm{Mn}^{2+}$ complexes of $\mathrm{N}$-ras p21, J. Chem. Phys., 98 (1993) 6704-6721.

[40] N.P. Benetis, P.C. Dave, D. Goldfarb, Characteristics of ESEEM and HYSCORE spectra of $S>1 / 2$ centers in orientationally disordered systems, J. Magn. Reson., 158 (2002) 126-142.

[41] F.C. Grozema, C. Houarner-Rassin, P. Prins, L.D.A. Siebbeles, H.L. Anderson, Supramolecular Control of Charge Transport in Molecular Wires, J. Am. Chem. Soc., 129 (2007) 13370-13371.

[42] S. van Doorslaer, G. Sierra, A. Schweiger, Dead time- dependent line distortions in absolute-value electron spin echo envelope modulation spectra, J. Magn. Reson., 136 (1999) 152-158.

[43] S. Stoll, A. Schweiger, EasySpin, a comprehensive software package for spectral simulation and analysis in EPR, J. Magn. Reson., 178 (2006) 42-55.

[44] A.M. Tyryshkin, S.A. Dikanov, D. Goldfarb, Sum Combination Harmonics in 4-pulse ESEEM Spectra Study of the ligand geometry in aqua vanadyl complexes in polycrystalline and glass matrices, J. Magn. Reson., Ser A, 105 (1993) 271-283.

[45] A.A. Shubin, S.A. Dikanov, The Influence of Nuclear Quadrupole Interactions upon Electron Spin-Echo Modulation Induced by Weak Hyperfine Interactions, J. Magn. Reson., 52 (1983) 1-12.

[46] C.P. Slichter, Principles of Magnetic Resonance, Springer Verlag, Berlin, 1980.

[47] S.A. Dikanov, L. Xun, A.B. Karpiel, A.M. Tyryshkin, M.K. Bowman, Orientationally-Selected TwoDimensional ESEEM Spectroscopy of the Rieske-Type Iron-Sulfur Cluster in 2, 4, 5-Trichlorophenoxyacetate Monooxygenase from Burkholderia cepacia AC1100, J. Am. Chem. Soc., 118 (1996) 8408-8416.

[48] B. Corzilius, E. Ramić, K.-P. Dinse, HYSCORE Analysis of Nitrogen Hyperfine Interactions, Appl. Magn. Reson., 30 (2006) 499-512.

[49] A.G. Maryasov, M.K. Bowman, Hyperfine Sublevel Correlation (HYSCORE) Spectra for Paramagnetic Centers with Nuclear Spin I=1 Having Isotropic Hyperfine Interactions, J. Phys. Chem. B, 108 (2004) 9412-9420.

[50] A.G. Maryasov, M.K. Bowman, Two-dimensional hyperfine sublevel correlation spectroscopy: Powder features for $S=1 / 2$, I=1, J. Magn. Reson., 179 (2006) 120135.

[51] F. Lendzian, R. Bittl, A. Telfer, W. Lubitz, Hyperfine structure of the photoexcited triplet state ${ }^{3} \mathrm{P} 680$ in plant PS II reaction centres as determined by pulse ENDOR spectroscopy, Biochim. Biophys. Acta, 1605 (2003) 3546.

[52] M. Thurnauer, ESR study of the photoexcited triplet state in photosynthetic bacteria, Reviews of Chemical Intermediates, 3 (1979) 197-230.

[53] J. Harmer, S. van Doorslaer, I.A. Gromov, A. Schweiger, Corrin nitrogens and remote dimethylbenzimidazole nitrogen interactions in Cob(II)alamin studied with HYSCORE at X- and Q-band, Chem. Phys. Lett., 358 (2002) 8-16.

[54] G. Jeschke, R. Rakhmatullin, A. Schweiger, Sensitivity Enhancement by Matched Microwave Pulses in One- and Two-Dimensional Electron Spin Echo Envelope Modulation Spectroscopy, J. Magn. Reson., 131 (1998) 261-271.

[55] S. Van Doorslaer, G. Jeschke, B. Epel, D. Goldfarb, R.-A. Eichel, B. Kräutler, A. Schweiger, Axial Solvent Coordination in "Base-Off" $\mathrm{Cob}(\mathrm{II})$ alamin and Related Co(II)-Corrinates Revealed by 2D-EPR, J. Am. Chem. Soc., 125 (2003) 5915-5927.

[56] C.E. Tait, P. Neuhaus, H.L. Anderson, C.R. Timmel, manuscript in preparation. 


\section{Appendix}

List of the coefficients used in equation 2 of the main text:

$$
\begin{aligned}
& \chi_{0}=1-\frac{k}{16}\left\{10+2 \gamma_{-}-4 \cos \omega_{12} \tau-4 \cos \omega_{34} \tau-\gamma_{-}\left[(1-\sqrt{1-k}) \cos \left(\omega_{12}+\omega_{34}\right) \tau-(1+\sqrt{1-k}) \cos \left(\omega_{12}-\omega_{34}\right) \tau\right]\right\} \\
& \chi_{d}=\frac{k}{4} \gamma_{+} \cos \left[\omega_{12}\left(t_{1}+t_{2}+\tau\right)\right]\left(1-\cos \omega_{34} \tau\right) \\
& \chi_{12}=\frac{k}{8}\left[2 \cos \left(\frac{\omega_{12} \tau}{2}\right)-(\sqrt{1-k}-1) \cos \left(\omega_{34} \tau+\frac{\omega_{12} \tau}{2}\right)+(\sqrt{1-k}+1) \cos \left(\omega_{34} \tau-\frac{\omega_{12} \tau}{2}\right)\right] \\
& \chi_{34}=\frac{k}{8}\left[2 \cos \left(\frac{\omega_{34} \tau}{2}\right)-(\sqrt{1-k}-1) \cos \left(\omega_{12} \tau+\frac{\omega_{34} \tau}{2}\right)+(\sqrt{1-k}+1) \cos \left(\omega_{12} \tau-\frac{\omega_{34} \tau}{2}\right)\right] \\
& \chi_{12,34}^{ \pm}=-\frac{k}{4}(\sqrt{1-k} \pm 1) \sin \left(\frac{\omega_{12} \tau}{2}\right) \sin \left(\frac{\omega_{34} \tau}{2}\right)
\end{aligned}
$$

List of the coefficients used in equation 9 of the main text:

$$
\begin{aligned}
& \chi_{0}=1-\gamma_{+} C_{0}-\gamma_{-} C_{1} \\
& +\left(\gamma_{+} C_{2}+\gamma_{-} C_{3}\right)\left[\cos \omega_{12} \tau+\cos \omega_{23} \tau+\cos \omega_{45} \tau+\cos \omega_{56} \tau\right]+\left(3 \gamma_{+} C_{4}+\gamma_{-} C_{5}\right)\left[\cos \omega_{13} \tau+\cos \omega_{46} \tau\right] \\
& +\gamma_{-}\left[C_{6}^{+}\left[\cos \left(\omega_{12}+\omega_{45}\right) \tau+\cos \left(\omega_{23}+\omega_{56}\right) \tau\right]+C_{6}^{-}\left[\cos \left(\omega_{12}-\omega_{56}\right) \tau+\cos \left(\omega_{23}-\omega_{45}\right) \tau\right]\right. \\
& +C_{7}^{+}\left[\cos \left(\omega_{12}+\omega_{56}\right) \tau+\cos \left(\omega_{23}+\omega_{45}\right) \tau\right]+C_{7}^{-}\left[\cos \left(\omega_{12}-\omega_{45}\right) \tau+\cos \left(\omega_{23}-\omega_{56}\right) \tau\right] \\
& +C_{8}^{+}\left[\cos \left(\omega_{13}+\omega_{45}\right) \tau+\cos \left(\omega_{13}+\omega_{56}\right) \tau+\cos \left(\omega_{12}+\omega_{46}\right) \tau+\cos \left(\omega_{23}+\omega_{46}\right) \tau\right] \\
& +C_{8}^{-}\left[\cos \left(\omega_{13}-\omega_{45}\right) \tau+\cos \left(\omega_{13}-\omega_{56}\right) \tau+\cos \left(\omega_{12}-\omega_{46}\right) \tau+\cos \left(\omega_{23}-\omega_{46}\right) \tau\right] \\
& \left.+C_{9}^{+} \cos \left(\omega_{13}+\omega_{46}\right) \tau+C_{9}^{-} \cos \left(\omega_{13}-\omega_{46}\right) \tau\right] \\
& \chi_{d}=\gamma_{+}\left[\cos \left[\omega_{12}\left(t_{1}+t_{2}+\tau\right)\right]\left[C_{2}-C_{16}^{+} \cos \omega_{45} \tau-C_{16}^{-} \cos \omega_{56} \tau-2 C_{4} \cos \omega_{46} \tau\right]\right. \\
& +\cos \left[\omega_{45}\left(t_{1}+t_{2}+\tau\right)\right]\left[C_{2}-C_{16}^{+} \cos \omega_{12} \tau-C_{16}^{-} \cos \omega_{23} \tau-2 C_{4} \cos \omega_{13} \tau\right] \\
& +\cos \left[\omega_{23}\left(t_{1}+t_{2}+\tau\right)\right]\left[C_{2}-C_{16}^{-} \cos \omega_{45} \tau-C_{16}^{+} \cos \omega_{56} \tau-2 C_{4} \cos \omega_{46} \tau\right] \\
& +\cos \left[\omega_{56}\left(t_{1}+t_{2}+\tau\right)\right]\left[C_{2}-C_{16}^{-} \cos \omega_{12} \tau-C_{16}^{+} \cos \omega_{23} \tau-2 C_{4} \cos \omega_{13} \tau\right] \\
& +\cos \left[\omega_{13}\left(t_{1}+t_{2}+\tau\right)\right]\left[3 C_{4}-2 C_{4} \cos \omega_{45} \tau-2 C_{4} \cos \omega_{56} \tau+C_{4} \cos \omega_{46} \tau\right] \\
& \left.+\cos \left[\omega_{46}\left(t_{1}+t_{2}+\tau\right)\right]\left[3 C_{4}-2 C_{4} \cos \omega_{12} \tau-2 C_{4} \cos \omega_{23} \tau+C_{4} \cos \omega_{13} \tau\right]\right] \\
& \chi_{12}=2 C_{3} \cos \frac{\omega_{12} \tau}{2}+C_{10} \cos \left(\frac{\omega_{12} \tau}{2}+\omega_{23} \tau\right)-4 C_{11}^{+} \cos \left(\frac{\omega_{12} \tau}{2}+\omega_{45} \tau\right)-4 C_{11}^{-} \cos \left(\frac{\omega_{12} \tau}{2}-\omega_{56} \tau\right) \\
& -C_{12}^{+} \cos \left(\frac{\omega_{12} \tau}{2}-\omega_{45} \tau\right)-C_{12}^{-} \cos \left(\frac{\omega_{12} \tau}{2}+\omega_{56} \tau\right)-C_{13}^{+} \cos \left(\frac{\omega_{12} \tau}{2}+\omega_{46} \tau\right)-C_{13}^{-} \cos \left(\frac{\omega_{12} \tau}{2}-\omega_{46} \tau\right) \\
& -C_{11}^{+}\left[2 \cos \left(\frac{\left(\omega_{13}+\omega_{23}\right) \tau}{2}-\omega_{45} \tau\right)-\cos \left(\frac{\left(\omega_{13}+\omega_{23}\right) \tau}{2}-\omega_{56} \tau\right)\right] \\
& -C_{11}^{-}\left[2 \cos \left(\frac{\left(\omega_{13}+\omega_{23}\right) \tau}{2}+\omega_{56} \tau\right)-\cos \left(\frac{\left(\omega_{13}+\omega_{23}\right) \tau}{2}+\omega_{45} \tau\right)\right] \\
& -2 C_{9}^{+} \cos \left(\frac{\left(\omega_{13}+\omega_{23}\right) \tau}{2}+\omega_{46} \tau\right)-2 C_{9}^{-} \cos \left(\frac{\left(\omega_{13}+\omega_{23}\right) \tau}{2}-\omega_{46} \tau\right)
\end{aligned}
$$




$$
\begin{aligned}
& \chi_{13}=2 C_{5} \cos \frac{\omega_{13} \tau}{2}+C_{10} \cos \left(\frac{\omega_{13} \tau}{2}-\omega_{23} \tau\right)-2 C_{9}^{-} \cos \left(\frac{\omega_{13} \tau}{2}+\omega_{46} \tau\right)+2 C_{9}^{+} \cos \left(\frac{\omega_{13} \tau}{2}-\omega_{46} \tau\right) \\
& -C_{13}^{+}\left[\cos \left(\frac{\omega_{13} \tau}{2}+\omega_{45} \tau\right)+\cos \left(\frac{\omega_{13} \tau}{2}+\omega_{56} \tau\right)\right]-C_{13}^{-}\left[\cos \left(\frac{\omega_{13} \tau}{2}-\omega_{45} \tau\right)+\cos \left(\frac{\omega_{13} \tau}{2}-\omega_{56} \tau\right)\right] \\
& -C_{14}\left[2 \cos \left(\frac{\left(\omega_{12}-\omega_{23}\right) \tau}{2}+\omega_{45} \tau\right)+2 \cos \left(\frac{\left(\omega_{12}-\omega_{23}\right) \tau}{2}-\omega_{56} \tau\right)-\cos \left(\frac{\left(\omega_{12}-\omega_{23}\right) \tau}{2}+\omega_{46} \tau\right)-\cos \left(\frac{\left(\omega_{12}-\omega_{23}\right) \tau}{2}-\omega_{46} \tau\right)\right] \\
& -C_{15}\left[\cos \left(\frac{\left(\omega_{12}-\omega_{23}\right) \tau}{2}-\omega_{45} \tau\right)+\cos \left(\frac{\left(\omega_{12}-\omega_{23}\right) \tau}{2}+\omega_{56} \tau\right)\right] \\
& \chi_{12,45}^{+}=2 C_{7}^{-} \cos \left(\frac{\left(\omega_{12}-\omega_{45}\right) \tau}{2}\right)-C_{12}^{+} \cos \left(\frac{\left(\omega_{12}+\omega_{45}\right) \tau}{2}\right) \\
& -C_{11}^{+}\left[\cos \left(\frac{\left(\omega_{13}+\omega_{23}-\omega_{45}\right) \tau}{2}\right)+\cos \left(\frac{\left(\omega_{12}-\omega_{46}-\omega_{56}\right) \tau}{2}\right)\right] \\
& -C_{15}\left[\cos \left(\frac{\left(\omega_{13}+\omega_{23}+\omega_{45}\right) \tau}{2}\right)+\cos \left(\frac{\left(\omega_{12}+\omega_{46}+\omega_{56}\right) \tau}{2}\right)\right] \\
& +C_{11}^{-} \cos \left(\frac{\left(\omega_{13}+\omega_{23}+\omega_{46}+\omega_{56}\right) \tau}{2}\right) \\
& \chi_{12,56}^{+}=2 C_{6}^{-} \cos \left(\frac{\left(\omega_{12}-\omega_{56}\right) \tau}{2}\right)-4 C_{11}^{-} \cos \left(\frac{\left(\omega_{12}+\omega_{56}\right) \tau}{2}\right) \\
& -2 C_{14}\left[\cos \left(\frac{\left(\omega_{12}+\omega_{46}+\omega_{45}\right) \tau}{2}\right)+\cos \left(\frac{\left(\omega_{13}+\omega_{23}+\omega_{56}\right) \tau}{2}\right)\right] \\
& -2 C_{11}^{+}\left[\cos \left(\frac{\left(\omega_{12}-\omega_{46}-\omega_{45}\right) \tau}{2}\right)+\cos \left(\frac{\left(\omega_{13}+\omega_{23}-\omega_{56}\right) \tau}{2}\right)\right] \\
& +4 C_{9}^{-} \cos \left(\frac{\left(\omega_{13}+\omega_{23}-\omega_{45}-\omega_{46}\right) \tau}{2}\right) \\
& \chi_{12,46}^{+}=2 C_{8}^{-} \cos \left(\frac{\left(\omega_{12}-\omega_{46}\right) \tau}{2}\right)-C_{13}^{-} \cos \left(\frac{\left(\omega_{12}+\omega_{46}\right) \tau}{2}\right) \\
& +C_{14} \cos \left(\frac{\left(\omega_{13}+\omega_{23}+\omega_{46}\right) \tau}{2}\right)-2 C_{9}^{-} \cos \left(\frac{\left(\omega_{13}+\omega_{23}-\omega_{46}\right) \tau}{2}\right) \\
& +C_{11}^{+}\left[\cos \left(\frac{\left(\omega_{12}-\omega_{45}+\omega_{56}\right) \tau}{2}\right)-2 \cos \left(\frac{\left(\omega_{12}+\omega_{45}-\omega_{56}\right) \tau}{2}\right)\right. \\
& \left.+\cos \left(\frac{\left(\omega_{13}+\omega_{23}+\omega_{45}-\omega_{56}\right) \tau}{2}\right)\right] \\
& \chi_{13,46}^{+}=2 C_{9}^{-}\left[\cos \left(\frac{\left(\omega_{13}+\omega_{46}\right) \tau}{2}\right)+\cos \left(\frac{\left(\omega_{13}-\omega_{46}\right) \tau}{2}\right)\right. \\
& -\cos \left(\frac{\left(\omega_{12}-\omega_{23}+\omega_{46}\right) \tau}{2}\right)-\cos \left(\frac{\left(\omega_{12}-\omega_{23}-\omega_{46}\right) \tau}{2}\right) \\
& -\cos \left(\frac{\left(\omega_{13}+\omega_{45}-\omega_{56}\right) \tau}{2}\right)-\cos \left(\frac{\left(\omega_{13}-\omega_{45}+\omega_{56}\right) \tau}{2}\right) \\
& \left.+2 \cos \left(\frac{\left(\omega_{12}-\omega_{23}+\omega_{45}-\omega_{56}\right) \tau}{2}\right)\right] \\
& \chi_{12,45}^{-}=2 C_{6}^{+} \cos \left(\frac{\left(\omega_{12}+\omega_{45}\right) \tau}{2}\right)-4 C_{11}^{+} \cos \left(\frac{\left(\omega_{12}-\omega_{45}\right) \tau}{2}\right) \\
& -2 C_{11}^{-}\left[\cos \left(\frac{\left(\omega_{13}+\omega_{23}+\omega_{45}\right) \tau}{2}\right)+\cos \left(\frac{\left(\omega_{12}+\omega_{46}+\omega_{56}\right) \tau}{2}\right)\right] \\
& -2 C_{14}\left[\cos \left(\frac{\left(\omega_{13}+\omega_{23}-\omega_{45}\right) \tau}{2}\right)+\cos \left(\frac{\left(\omega_{12}-\omega_{46}-\omega_{56}\right) \tau}{2}\right)\right] \\
& +4 C_{9}^{+} \cos \left(\frac{\left(\omega_{13}+\omega_{23}+\omega_{46}+\omega_{56}\right) \tau}{2}\right) \\
& \chi_{12,56}^{-}=2 C_{7}^{+} \cos \left(\frac{\left(\omega_{12}+\omega_{56}\right) \tau}{2}\right)-C_{12}^{-} \cos \left(\frac{\left(\omega_{12}-\omega_{56}\right) \tau}{2}\right) \\
& +C_{11}^{-}\left[\cos \left(\frac{\left(\omega_{12}+\omega_{46}+\omega_{45}\right) \tau}{2}\right)+\cos \left(\frac{\left(\omega_{13}+\omega_{23}+\omega_{56}\right) \tau}{2}\right)\right] \\
& -C_{15}\left[\cos \left(\frac{\left(\omega_{12}-\omega_{46}-\omega_{45}\right) \tau}{2}\right)+\cos \left(\frac{\left(\omega_{13}+\omega_{23}-\omega_{56}\right) \tau}{2}\right)\right] \\
& +C_{11}^{+} \cos \left(\frac{\left(\omega_{13}+\omega_{23}-\omega_{45}-\omega_{46}\right) \tau}{2}\right) \\
& \chi_{12,46}^{-}=2 C_{8}^{+} \cos \left(\frac{\left(\omega_{12}+\omega_{46}\right) \tau}{2}\right)-C_{13}^{+} \cos \left(\frac{\left(\omega_{12}-\omega_{46}\right) \tau}{2}\right) \\
& -2 C_{9}^{+} \cos \left(\frac{\left(\omega_{13}+\omega_{23}+\omega_{46}\right) \tau}{2}\right)+C_{14} \cos \left(\frac{\left(\omega_{13}+\omega_{23}-\omega_{46}\right) \tau}{2}\right) \\
& +C_{11}^{-}\left[\cos \left(\frac{\left(\omega_{12}-\omega_{45}+\omega_{56}\right) \tau}{2}\right)-2 \cos \left(\frac{\left(\omega_{12}+\omega_{45}-\omega_{56}\right) \tau}{2}\right)\right. \\
& \left.+\cos \left(\frac{\left(\omega_{13}+\omega_{23}+\omega_{45}-\omega_{56}\right) \tau}{2}\right)\right] \\
& \chi_{13,46}^{-}=2 C_{9}^{+}\left[\cos \left(\frac{\left(\omega_{13}+\omega_{46}\right) \tau}{2}\right)+\cos \left(\frac{\left(\omega_{13}-\omega_{46}\right) \tau}{2}\right)\right. \\
& -\cos \left(\frac{\left(\omega_{12}-\omega_{23}+\omega_{46}\right) \tau}{2}\right)-\cos \left(\frac{\left(\omega_{12}-\omega_{23}-\omega_{46}\right) \tau}{2}\right) \\
& -\cos \left(\frac{\left(\omega_{13}+\omega_{45}-\omega_{56}\right) \tau}{2}\right)-\cos \left(\frac{\left(\omega_{13}-\omega_{45}+\omega_{56}\right) \tau}{2}\right) \\
& \left.+2 \cos \left(\frac{\left(\omega_{12}-\omega_{23}+\omega_{45}-\omega_{56}\right) \tau}{2}\right)\right]
\end{aligned}
$$




$$
\begin{array}{lll}
C_{0}=k\left(\frac{2}{3}-\frac{3}{8} k\right) & C_{6}^{ \pm}=\frac{k^{2}}{16}\left(1 \pm \frac{1}{3} \sqrt{1-k}-\frac{5}{6} k\right) & C_{12}^{ \pm}=\frac{k}{6}\left(1 \pm \sqrt{1-k}-\frac{13}{8} k \mp \frac{7}{8} k \sqrt{1-k}+\frac{5}{8} k^{2}\right) \\
C_{1}=k\left(1-\frac{11}{16} k+\frac{3}{32} k^{2}\right) & C_{7}^{ \pm}=\frac{k}{24}(1 \mp \sqrt{1-k}-k)^{2} & C_{13}^{ \pm}=\frac{k^{2}}{96}(2 \pm 2 \sqrt{1-k}+k) \\
C_{2}=\frac{k}{2}\left(\frac{1}{3}-\frac{1}{4} k\right) & C_{8}^{ \pm}=\frac{k^{2}}{48}\left(1 \mp \sqrt{1-k}-\frac{1}{4} k\right) & C_{14}=\frac{1}{96} k^{3} \\
C_{3}=\frac{k}{2}\left(\frac{1}{3}-\frac{1}{3} k+\frac{1}{16} k^{2}\right) & C_{9}^{ \pm}=\frac{k^{2}}{192}(1 \mp \sqrt{1-k})^{2} & C_{15}=\frac{k^{2}}{24}(1-k) \\
C_{4}=\frac{1}{48} k^{2} & C_{10}=\frac{k^{2}}{2}\left(\frac{1}{3}-\frac{1}{4} k\right) & C_{16}^{ \pm}=\frac{k}{12}(1 \pm \sqrt{1-k}-k) \\
C_{5}=\frac{k^{2}}{16}\left(\frac{1}{3}+\frac{1}{2} k\right) & C_{11}^{ \pm}=\frac{k^{2}}{48}(1 \pm \sqrt{1-k}-k) &
\end{array}
$$

The remaining coefficients $\chi$ can be derived from the ones given above by substitution of the frequency indices according to the following table:

$\begin{array}{ccc}\text { Coefficient } & \text { Initial Coefficient } & \text { Substitution of frequency indice } \\ \chi_{23} & \chi_{12} & 12 \leftrightarrow 23,45 \leftrightarrow 56 \\ \chi_{45} & \chi_{12} & 12 \leftrightarrow 45,23 \leftrightarrow 56,13 \leftrightarrow 46 \\ \chi_{56} & \chi_{12} & 12 \leftrightarrow 56,23 \leftrightarrow 45,13 \leftrightarrow 46 \\ \chi_{46} & \chi_{13} & 12 \leftrightarrow 45,23 \leftrightarrow 56,13 \leftrightarrow 46 \\ & & \\ \chi_{23,45}^{+} & \chi_{12,56}^{+} & 12 \leftrightarrow 23,45 \leftrightarrow 56 \\ \chi_{23,45}^{-} & \chi_{12,56}^{-} & 12 \leftrightarrow 23,45 \leftrightarrow 56 \\ \chi_{23,56}^{+} & \chi_{12,45}^{+} & 12 \leftrightarrow 23,45 \leftrightarrow 56 \\ \chi_{23,56}^{-} & \chi_{12,45}^{-} & 12 \leftrightarrow 23,45 \leftrightarrow 56 \\ \chi_{23,46}^{+} & \chi_{12,46}^{+} & 12 \leftrightarrow 23,45 \leftrightarrow 56 \\ \chi_{23,46}^{-} & \chi_{12,46}^{-} & 12 \leftrightarrow 45,23 \leftrightarrow 56,13 \leftrightarrow 46 \\ \chi_{13,45}^{+} & \chi_{12,46}^{+} & 12 \leftrightarrow 45,23 \leftrightarrow 56,13 \leftrightarrow 46 \\ \chi_{13,45}^{-} & \chi_{12,46}^{-} & 12 \leftrightarrow 56,23 \leftrightarrow 45,13 \leftrightarrow 46 \\ \chi_{13,56}^{+} & \chi_{12,46}^{+} & 12 \leftrightarrow 56,23 \leftrightarrow 45,13 \leftrightarrow 46 \\ \chi_{13,56}^{-} & \chi_{12,46}^{-} & \end{array}$

